\title{
A Method Combining Fractal Analysis and Single Channel ICA for Vibration Noise Reduction
}

\author{
Quanbo Lu (i) and Mei Li (i) \\ School of Information Engineering, China University of Geosciences, Beijing 100083, China \\ Correspondence should be addressed to Mei Li; maggieli@cugb.edu.cn
}

Received 2 March 2021; Revised 19 April 2021; Accepted 3 May 2021; Published 17 May 2021

Academic Editor: Marcello Vanali

Copyright (C) 2021 Quanbo Lu and Mei Li. This is an open access article distributed under the Creative Commons Attribution License, which permits unrestricted use, distribution, and reproduction in any medium, provided the original work is properly cited.

\begin{abstract}
Aiming at the problem that real engineering vibration signals are interfered by strong noise, this paper proposes a method combining single channel-independent component analysis (SCICA) and fractal analysis (FD) to reduce the effect of noise on the time-frequency analysis of vibration signals. First, phase space reconstruction is performed on the vibration signal to make the proper input for ICA algorithm. The original is then decomposed into several component signals. The fractal dimension of each component signals is calculated to determine whether the signal should be considered noise. Noisy component signals are then processed by wavelet denoising. Finally, the output signal after noise reduction is reconstructed using the filtered "right" component signals. This paper uses the method to analyze real noisy vibration signal. Experimental results show the effectiveness of the proposed method.
\end{abstract}

\section{Introduction}

The measured vibration signals contain many high-frequency and low-frequency noises because of the test environment. Thus, the spectral components of the signal are complex. In addition, extracting the envelope of the signal is difficult for the reason of noise interference. Noise causes the inaccurate decomposition of the signal envelope, particularly, when using time-frequency analysis technology. An effective noise reduction method for time-frequency analysis would improve time-frequency resolution and algorithm performance, which are required both for practical applications and theoretical analysis. Wavelet denoising is a vibration signal, Qin et al. used the wavelet denoising method to improving the detection performance of distributed vibration sensor [1], and Xie et al. proposed an improved wavelet denoise method for vibration signal processing [2]. Other methods proposed an improved EMD method for time-frequency feature extraction of telemetry vibration [3]. $\mathrm{Li}$ et al. proposed a novel noise reduction technique for underwater acoustic signals based on complete ensemble empirical mode decomposition with adaptive noise, minimum mean square variance criterion, and least mean square adaptive filter [4]. For warship radio noise, Li et al. proposed a novel linear spectrum frequency feature extraction technique for warship radio noise based on complete ensemble empirical mode decomposition with adaptive noise, duffing chaotic oscillator, and weighted-permutation entropy [5]. Wang et al. proposed a new fault diagnosis method based on adaptive spectrum mode extraction [6]. For the data-driven fault diagnosis, Wang et al. proposed a novel technique based on AlexNet Convolutional neural network [7].

ICA is a common blind source separation method but generally requires at least two input signals [8]. In 2001, James and Lowe proposed an algorithm based on a dynamic embedded framework to apply an ICA algorithm to extract multidimensional brain activity signals from single brain electromagnetic signals [9]. Davies and James suggested that SCICA can sometimes be used to separate important components from a time series [10]. And, in [11], the author reviewed the concept and current practice of BSS based on second-order statistics and on higher-order statistics, which is known as ICA [12], and applied ensemble empirical mode 
decomposition to make use of ICA on single-channel neuromuscular signals [13]. Guerrero has proposed an adaptive noise reduction method that combines ICA and recursive least square (RLS) for processing EEG signals [14]. However, this method filters all source signals after ICA decomposition. In other words, part of the useful signal will be likely filtered out as noise, which is unacceptable for vibration signal processing. If useful information is lost because of filtering, the analysis and identification of vibration signals will be ambiguous.

To solve this problem, this paper proposes a vibration signal noise reduction method based on SCICA and FD. First, the Newton iteration ICA method is performed using real vibration signal based on kurtosis maximization of the vibration signal for blind source separation. Second, the component signal required for filtering is selected using fractal dimensions, different denoising methods are performed on the selected component signals, and finally, the signals are reconstructed. The results show that the proposed algorithm not only greatly suppresses noise but also effectively avoids the loss of useful information.

\section{Methods}

Abnormal signal filtering is especially problematic because noise greatly reduces the vibration signals. If the method filters the entire source signal, some information will be lost. To address this problem, this paper uses the signals fractal dimension to constrain the ICA filtering process, avoiding the loss of useful information. As shown in Figure 1, the algorithm is described below:

(1) The collected vibration signal is preprocessed using zero drift correction and term trend elimination. Phase space reconstruction of the signal is then performed.

(2) Blind source separation is performed on the signal using kurtosis maximization via the Newton iterative ICA algorithm [15]. The source signal component $S=\left[\widehat{S}_{1}, \widehat{S}_{2}, \ldots, \widehat{S}_{M}\right]$ and separating matrix $W$ are then calculated.

(3) The grid dimension $D_{i}, i=1,2, \ldots, M$, and $M$ of each source signal are calculated. The source signal is screen filtered using the grid dimension. According to the preliminary analysis of many experiments, the grid dimension of source signals containing an abnormal signal is greater than 1.5. As a conservative screening condition, this paper sets the maximum step point of the grid dimension curve close to 1.5. Specific operational procedures: sequence grid dimension $D_{i}$ is in ascending order. The most significant step point of the arrangement curve is the screening conditions. The source signal component $\widehat{S}$ can be divided into one part $\widehat{S}_{f}$ to be filtered and the other part $\widehat{S}_{b}$ to be retained.

(4) Next, wavelet denoising is performed on the filtering source signal $\widehat{S}_{f f}$.

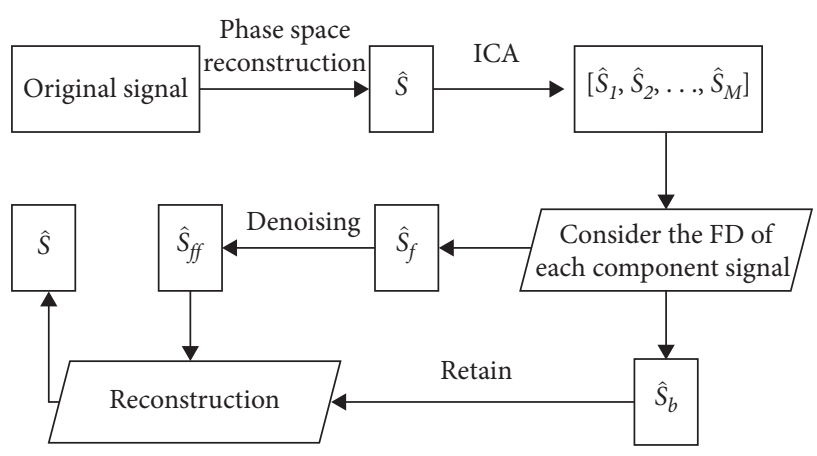

Figure 1: Total procedure.

(5) The filtered source signal $\widehat{S}_{f}$ and $\widehat{S}_{b}$ are combined to produce a recombinant source signal $\widehat{S}^{\prime}$. The same relative position is maintained during the combination. The separating matrix $W$ is used to reconstruct the phase space signal $X=W S^{\prime}$. The noise reduction vibration signal is obtained from $X$. A description of the phase space reduction method may be found elsewhere.

2.1. Phase Space Reconstruction. The phase space reconstruction method is required when using ICA blind source separation for processing the single-channel vector data. In this paper, the chaotic phase space reconstruction method is performed on the signal to extend to multidimensional phase space. Thus, each aliasing source signal is reopened in a multidimensional space, and the ICA method is used to separate the source signals. In the 1980s, Packard et al. proposed the use of chaotic time series to reconstruct the phase space to reveal the dynamics of the system [16]. The basic idea is that the evolution of any component in the system is related to the change of all interacting components. By the Takens embedding theory for one dimension signal, a phase space equivalent to the original dynamic system can be constructed by determining its embedding dimension and adding time delay. The phase space contains the required information and characteristics of the original signal to obtain enough hidden information in the observation signal. As the dynamic embedding principle, the phase space reconstruction method construct delay vectors by delaying one-dimensional observation signal, and combining these vectors to build a multidimensional phase space can reveal the dynamic system in the chaotic attractor and chaotic time series. In order to obtain enough information content, the reconstructed phase space embedding dimension $m$ should be at least greater than system freedom $D$, i.e., $m \geq 2 D+1$.

First, determine the embedding dimension $m$ and the time delay $\tau$; for a complex signal $x(t)$, the time delay $\tau$ is added to obtain the delay vector at a time $t$ :

$$
x(t)=[x(t), x(t+\tau), \ldots, x(t+(m-1) \tau)]^{T},
$$

where $t=1,2, \ldots, N-(m-1) \tau$ and $N$ is the length of the observed signal. These delay vectors are combined to form 
an embedding matrix, which is a multidimensional delay signal:

$$
x=\left(\begin{array}{cccc}
x_{1} & x_{2} & \cdots & x_{N-(m-1) \tau} \\
x_{1+\tau} & x_{2+\tau} & \cdots & x_{N-(m-1) \tau+\tau} \\
\vdots & \vdots & \ddots & \vdots \\
x_{1+(m-1) \tau} & x_{2+(m-1) \tau} & \cdots & x_{N}
\end{array}\right) .
$$

Without prior information about vibration signals, selecting an embedding dimension is difficult. Instead, a delay time is selected before determining the embedding dimension according to the delay time. Many algorithms may be used to calculate the delay time, including the C-C algorithm [17], G-P algorithm, and mutual information method [18]. The algorithm processes are detailed elsewhere. This paper compared the performance and computational efficiency and used the mutual information method to obtain the delay time. Finally, using the method proposed by Takens [19], the Cao algorithm [20] was implemented to calculate the embedding dimension.

\subsection{Newton Iteration ICA Algorithm Based on Kurtosis} Maximization. The phase space reconstruction expended the single-channel signal into multiple channels. Thus, ICA can be applied. The ICA algorithm is a blind source separation method that uses the assumption of statistical independence of alias components. A separating matrix $W$ can be constructed, and blind source separation can be realized as follows:

$$
\left\{\begin{array}{l}
X=A S, \\
\widehat{S}=W X,
\end{array}\right.
$$

where $X=\left[x_{1}, x_{2}, \ldots, x_{N}\right]^{T}$ is the aliasing signal vector, $A$ is the mixing matrix, $S=\left[S_{1}, S_{2}, \ldots, S_{M}\right]^{T}$ is the source signal vector, and $\widehat{S}=\left[\widehat{S}_{1}, \widehat{S}_{2}, \ldots, \widehat{S}_{M}\right]^{T}$ is the estimated value of $S$. The traditional method, which is based on ICA kurtosis and uses the fixed point iterative method to calculate the separating matrix, has many shortcomings, such as low computational efficiency, the possibility that the separating matrix fails to converge, and poor separation effects. Li et al. proposed an alternative method based on the Newton iteration to calculate the separating matrix [21]. However, the results of the algorithm are unstable when the mean of the source signal is zero. To address these problems, this paper uses Newton iteration ICA by maximizing the kurtosis. The constraint matrix separation operation using Newton iterative algorithm is based on the kurtosis formula. The source signals may be obtained by reducing the Gaussian of the separation results. Simulation results show that this method improves the computational efficiency and separation effects. First, the kurtosis of the signal is defined as follows [22]:

$$
\operatorname{kurt}(\widehat{s})=E\left(\widehat{s}^{4}\right)-3\left(E\left(\widehat{s}^{2}\right)\right)^{2} .
$$

Let $\widehat{s}=w^{T} x$, where $x$ and $w$ is a component of the separating matrix $W$. The gradient $\operatorname{kurt}(\widehat{s})$ of $w$ is

$$
\nabla_{w} \operatorname{kurt}\left(w^{T} x\right)=4 E\left(\left(w^{T} x\right)^{3}\right)-3 E\left(\left(w^{T} x\right)^{2}\right) .
$$

To maximize the kurtosis, the extreme solution is calculated under the constraints $E\left(w^{T} x^{2}\right)=\|w\|=1$ using the Lagrange algorithm as shown below:

$$
l=\operatorname{kurt}\left(w^{T} x\right)=4 E\left(\left(w^{T} x\right)^{3}\right)+1.5 E\left(w^{T} x^{2}\right)-1 .
$$

The iterative formula for $w$ is calculated using the Newton iterative method:

$$
w(k+1)=w(k)-\frac{E\left(x\left(w(k)^{T} x\right)^{3}\right)}{3 E\left(\left(w(k)^{T} x\right)^{2}\right)} .
$$

After each iteration, the decorrelation extraction and normalization are performed on the linear combination of independent components to prevent these components from converging to the same extreme point, which leads to the instability of the algorithm because of the zero-mean of the source signal. The formulas for this method are shown as follows:

$$
\begin{aligned}
& w_{p+1}(k+1)=w_{p+1}(k)-\frac{E\left(x\left(w_{p+1}(k)^{T} x\right)^{3}\right)}{3 E\left(\left(w_{p+1}(k)^{T} x\right)^{2}\right)}, \\
& w_{p+1}(k+1)=w_{p+1}(k+1)-\sum_{j=1}^{p} w_{p+1}(k+1)^{T} w_{j} w_{j}, \\
& w_{p+1}(k+1)=\frac{w_{p+1}(k+1)}{\sqrt{w_{p+1}(k+1)^{T} w_{p+1}(k+1)}} .
\end{aligned}
$$

The separating matrix $W$ is calculated by estimating all the components $w$ with the method described above.

2.3. Fractal Dimension. Fractal theory, an active area of signal processing research, is a mathematical tool that quantitatively describes various complex signals in nature. The fractal dimension of the vibration signal may be calculated to measure the complexity and irregularity of the signal. In fractal theory, larger fractal dimensions indicate that the signal is complex. For vibration signal processing, noise often causes the vibration signal components to be complicated, nonlinear, and nonstationary. Thus, the fractal dimension of the vibration signal is large. Many experimental results show the fractal dimension (grid dimension) of abnormal signals is greater than 1.5. Hence, the source signals with a larger fractal dimension may be filtered out avoiding meaningful information loss. The noise may be suppressed using a wavelet filter on the source signal, which has smaller fractal dimension. The fractal dimension contains the box dimension, correlation dimension, Hausdorff dimension, and grid dimension. For one-dimensional signals, the box dimension is equivalent to the grid dimension, which simplifies the computer processing. For an signal 
channel signal $\left\{x \mid x_{i}, i=1,2, \ldots, N\right\}$, the grid dimension is defined as

$$
D=-\frac{\ln \left(M_{j}\right)}{\ln (\Delta t)},
$$

where $\Delta t$ is the sampling interval, $M_{j}=\sum\left[x_{i+1-x_{i}} / \Delta t\right]$, and $[x]$ represents the closest integer that is greater than $x$. First, the space of discrete signals is divided into a multidimensional grid, and the grid dimension is calculated by counting the grid points. The Euclidean space of discrete signals $R^{N}$ is divided as small as possible into grids with the area of $\Delta$. Points' count $N_{\Delta K}$ of grid space of the signal $\left\{x \mid x_{i}, i=1,2, \ldots, N\right\}$ is calculated. Different areas $N_{\Delta K}$ are used to calculate the $N_{\Delta K}$ point, yielding a point set $\left\{x_{k}, y_{k}\right\}$ :

$$
\left\{\begin{array}{l}
x_{k}=-\ln \left(\Delta_{K}\right), \\
y_{k}=-\ln \left(N_{\Delta K}\right) .
\end{array}\right.
$$

The slope of the line formed by the set of points $\left\{x_{k}, y_{k}\right\}$ is the grid dimension of the signal. The line is not straight, so the least-squares method is often used to fit a line for practical applications. Finally, the grid dimension $D$ may be obtained.

\section{Experiments and Results}

This section contains 3 groups of experiments. The first uses general sin wave to show the effectiveness of the SCICA with phase space reconstruction. Two cases are given respective correspondence to the condition with or without noise. The section and third experiments apply the SCICA with FD (SCICA-FD) in noise reduction. Experiment 2 combines SCICA-FD with wavelet denoising, the basic knowledge of wavelet is given, and the experiment result is discussed. Similar to experiment 2, experiment 3 applies smoothing filtering to SCICA-FD to show the proposed method can be applied to different denoising methods.

\subsection{Validation of SCICA Based on Phase Space Reconstruction}

Case 1. Using general sine waves and performing phase space reconstruction-based SCICA to verify the effect, in the absence of noise, signal 1 and signal 2 and their mixed-signal are shown in Figure 2(a). SCICA firstly separates the mixedsignal with time delay calculated as 3 and the embedding dimension 7, which means 7 ICA components are obtained. Because too many signals are obtained by SCICA, principal component analysis (PCA) is applied to reduce the dimension to avoid choosing the signal from the 7 ICA output signals. It can be seen in Figure 2(b) that the three principal components with the largest contribution is added to more than $95 \%$, so PCA is used to reduce the 7 dimension signal matrix to three dimensions. From the result shown in Figure 2(c), it can be seen that the phase space reconstruction-based SCICA can effectively separate the two sine signals from the combined signal.
Case 2. Similar to Case 1, signal 1, signal 2, a random noise, and their mix signal are shown in Figure 3(a). The mixed signal is separated by SCICA with time delay which equals to 2 and embedding dimension which equals to 9 which means there is 9 ICA components. Again, PCA is used to reduce the dimension of the ICA output signal matrix. From Figure 3(b), the matrix should be reduced to 4 dimensions. The result is shown in Figure 3(c). It can be seen that compare to that of Case 1 (without noise), the output waveform has some fluctuation, but the signal obtained is still close enough to the original one.

3.2. Application to Wavelet Denoising. From a signal perspective, wavelet denoising is a signal filtering method. Although wavelet denoising can be viewed as a low pass filter, the signal features can be preserved successfully after denoising, so it is better than the traditional low-pass filter.

3.2.1. Necessary Knowledge of Wavelet Denoising. The model of a nonstationary, noisy, and one-dimensional signal is represented as follows:

$$
s_{i}=f_{i}+\sigma e_{i}, \quad i=0,1, \ldots, n-1,
$$

where $f_{i}$ is the true signal, $e_{i}$ is the noise, the noise property is unknown, and $s_{i}$ is the noisy signal series. To improve the signal-to-noise ratio (SNR) of the signal, it is necessary to reduce the noise. Taking the simplest noise model as an example, $e_{i}$ is considered as Gauss white noise $N(0,1)$. In the actual acquisition of vibration signal, the useful signal is usually low or some relatively stable signal and noise signal is normally high-frequency signal, and the noise reduction process can be processed according to the following methods. First, decompose the noise signal by wavelet transform, such as the three layers' decomposition, and the decomposition process is shown in Figure 4; the decomposed signals include two parts: approximate signal (CA3) and the detail signal (CD3, CD2, and CD1).

The approximate signal represents the decomposed lowfrequency signal. Detail signals stand for high-frequency part. Because the noise belongs to high frequency signals, it is usually included in each detail signal level. In the process, the approximate signal of wavelet transform is reserved, and the detail signal is processed in the form of a threshold value. After the above process, the signal is reconstructed by wavelet transform, thus obtains the denoised signal in which the useful part is well preserved.

The noise reduction process of the vibration signal $s$ can be divided into three steps:

Step 1 (vibration signal wavelet decomposition): select a wavelet, determine the wavelet decomposition layers $N$, and then take $N$ layer wavelet decomposition to signal s. Also, the appropriate choice of wavelet base has a significant impact on the noise reduction effect. A suitable wavelet basis can make the signal energy be distributed on a small number of substrates. 


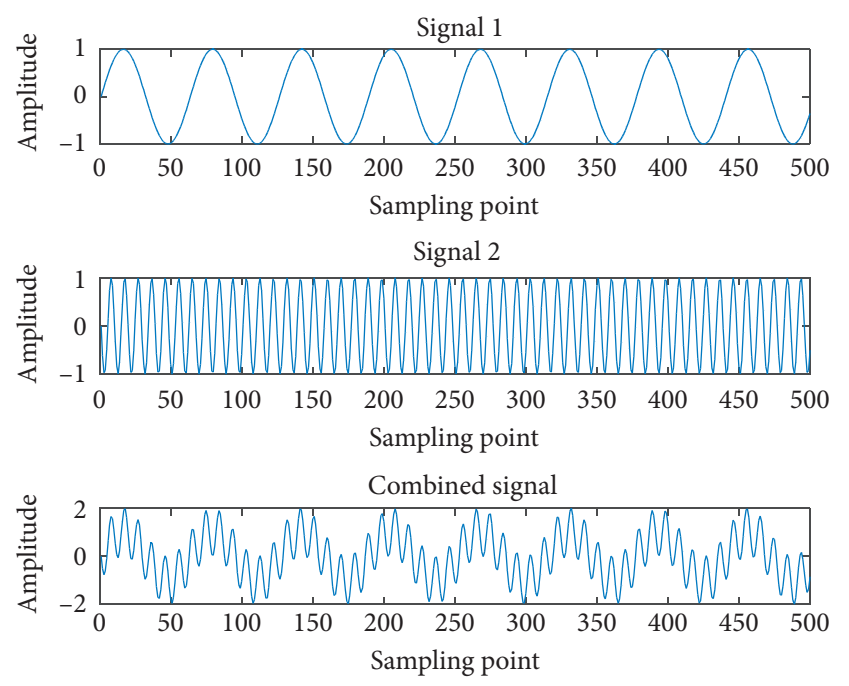

(a)

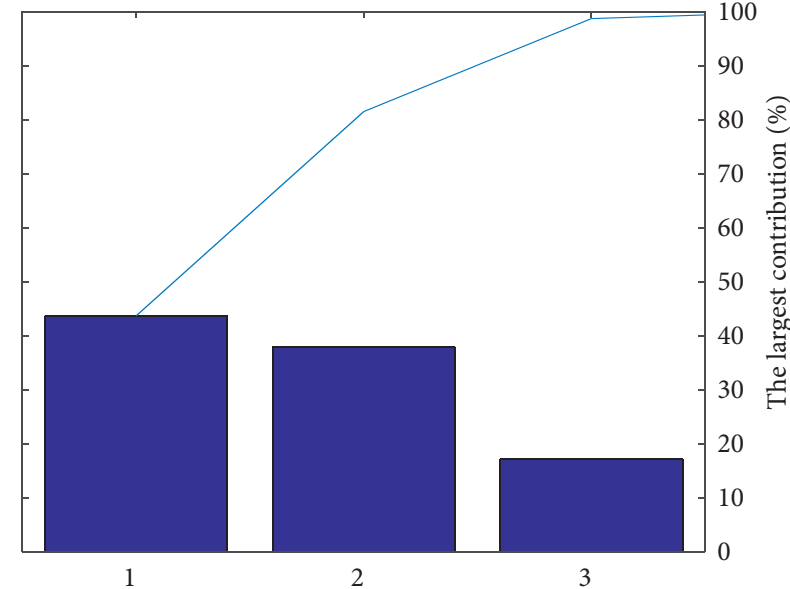

The principal component

(b)
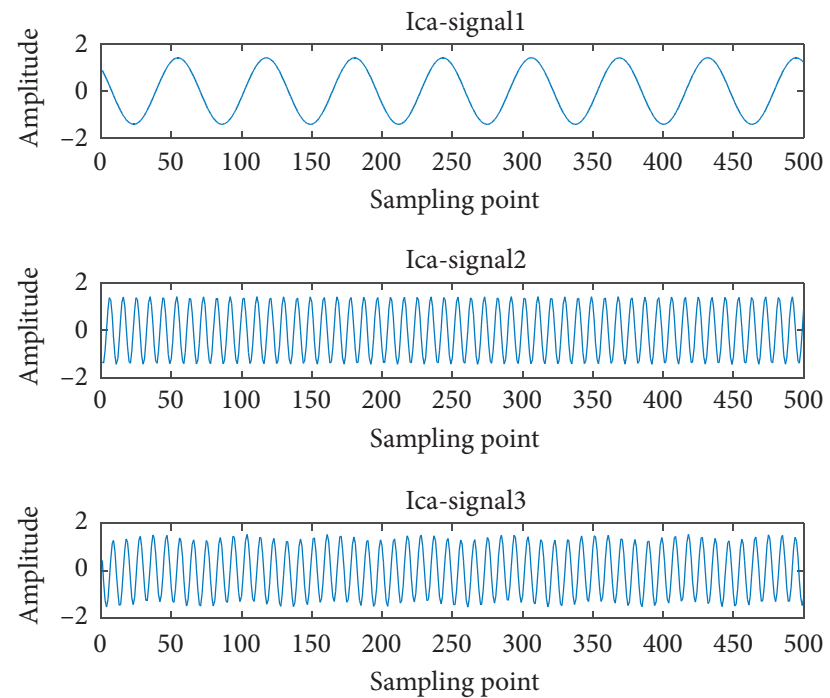

(c)

FIgure 2: Noise-free condition. (a) Original and combined signal, (b) PCA variance, and (c) separation results.

Step 2: after the wavelet decomposition, the high frequency coefficient's threshold value is quantized. The high-frequency coefficient of each layer of the first layer to the $\mathrm{N}$ layer is selected, and a threshold value is selected to process the soft threshold quantization.

Step 3 (one-dimensional wavelet reconstruction): according to the low-frequency coefficients of the fourth layer of wavelet decomposition and the high-frequency coefficients of the first layer to the $N$ layer, the wavelet reconstruction of the vibration signal is carried out. In the process of noise reduction, the threshold value of the highfrequency coefficient is selected, and the threshold and the soft threshold are given in two forms of Donoho [23]. Usually, the hard threshold denoising will cause the loss of available information, so the engineering signal analysis is mostly based on the soft threshold denoising technique. The method described in this paper is also a soft thresholding.
3.2.2. SCICA-FD with Wavelet Denoising. Using Case Western Reserve University's bearing data, Figure 5(a) shows the vibration signal in time domain and its spectrum is shown in Figure 5(b). As shown in Figure 5(b), the vibration signal time domain contains large background noise, and the fault information is submerged in the noise.

Using the proposed method, the vibration data is separated by SCICA. The fractal dimension of each separated signal is shown in Figure 6. As mentioned before, this paper chooses signals with fractal dimension close to 1.5 , which is number 4 to 13 in Figure 6.

Then, the same wavelet threshold is applied to the selected signal. The spectrum of the restored signal is shown in Figure 7(b). Performing only the wavelet denoising, the result is shown in Figure 7(a). Comparing to Figure 7(a), Figure 7 (a) can tell that the proposed method reduces the noise and reserves the high-frequency part. Some of the high-frequency parts are a little weak. 


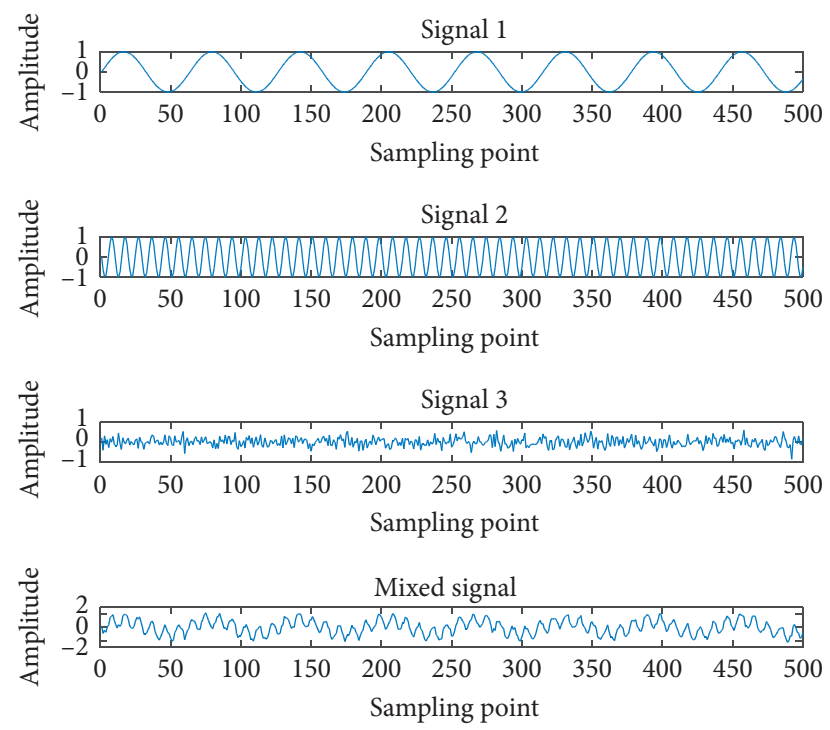

(a)

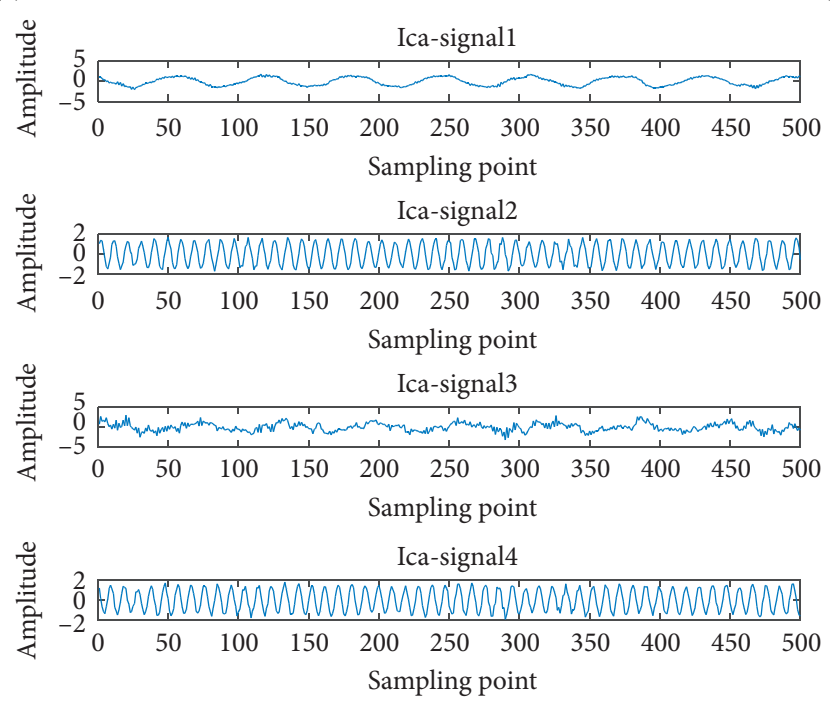

(c)

FIGURE 3: Noise added condition. (a) Original and combined signal, (b) PCA variance (with noise), and (c) separation results.

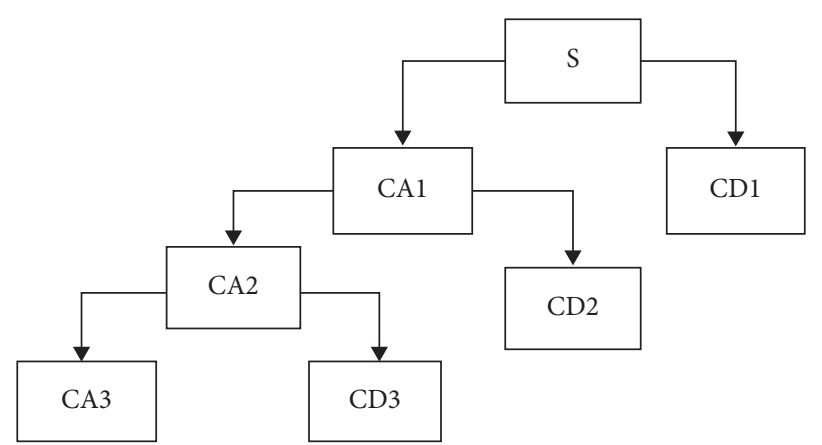

FIGURE 4: Sketch map of wave decomposition.

3.3. Application to Smooth Filtering. Wavelet denoising is applied in experiment 2; as mentioned, wavelet denoising can be considered a lowpass filter but retains more useful frequencies. The smoothing filter used here is called Savitzky-Golay filter which is proposed in 1964 [24]. Savitzky-Golay filter is a low-pass filter based on polynomial 


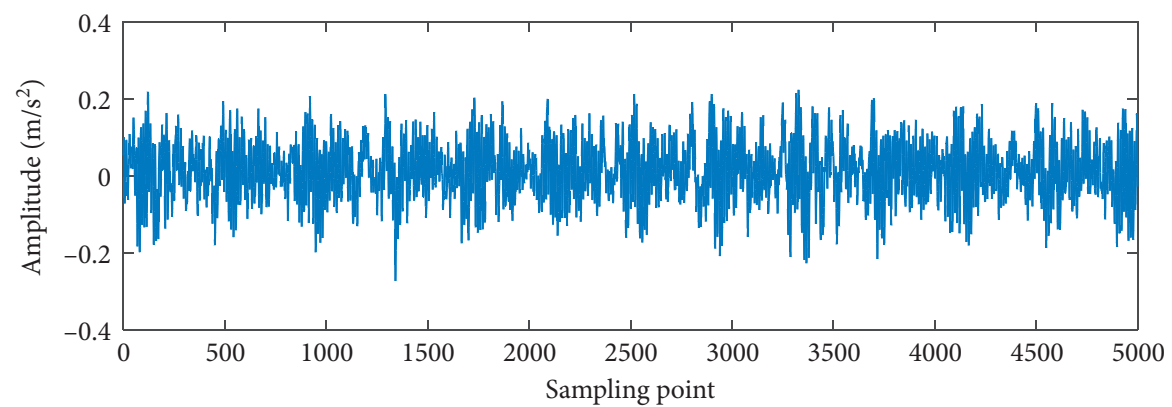

(a)

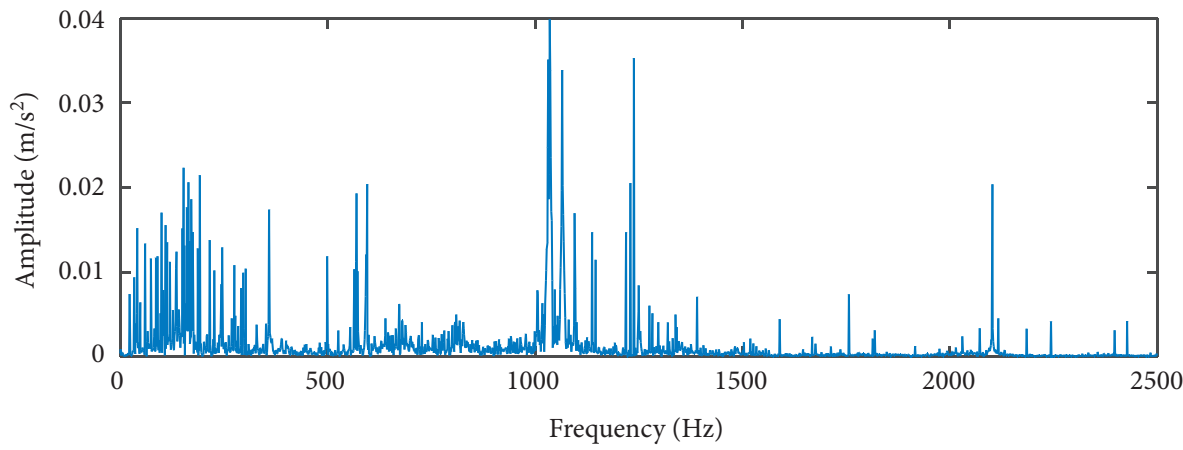

(b)

FIgURE 5: Vibration signal and its spectrum. (a) Time-domain waveform of vibration signal and (b) spectrum of the vibration signal.

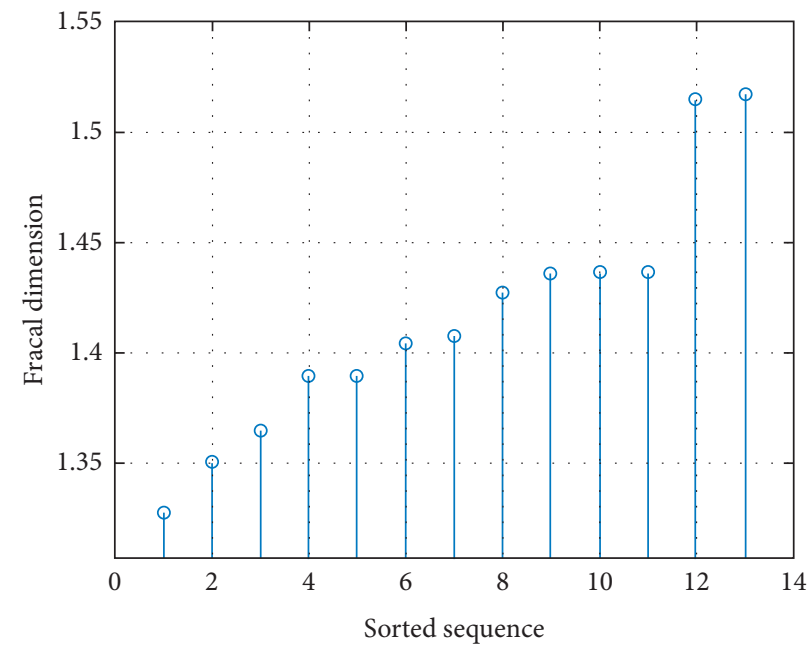

FIGURE 6: Fractal dimension of each ICA output signal.

fitting, compared to wavelet denoising, which is a simpler and more straightforward way. This paper shows in this part that our proposed SCICA-FD can be applied with even simpler methods.

3.3.1. Savitzky-Golay Filter. Let a set of data in $x(n)$ be $x(i)$, $i=M, \ldots, 0, \ldots, M$, and a polynomial of order $p$ is constructed:

$$
f_{i}=a_{0}+a_{1} i+\cdots+a_{p} i^{p}=\sum_{k=0}^{p} a_{k} i^{k}, \quad p \leq 2 M .
$$

The polynomial is used to fit the data. Due to the existence of fitting errors, the sum of squares of the total error is

$$
E=\sum_{-M}^{M}\left[f_{i}-x(i)\right]^{2}=\sum_{-M}^{M}\left[\sum_{k=0}^{p} a_{k} i^{k}-x(i)\right]^{2} .
$$

To minimize $E$, the derivative of $E$ to each coefficient is set to $0,\left(\partial_{E} / \partial_{a_{r}}\right)=0, r=0,1,2, \ldots, p$, that is,

$$
\frac{\partial_{E}}{\partial_{a_{r}}}=\frac{\partial\left[\sum_{-M}^{M}\left[\sum_{k=0}^{p} a_{k} i^{k}-x(i)\right]^{2}\right]}{\partial_{a_{r}}}=0,
$$




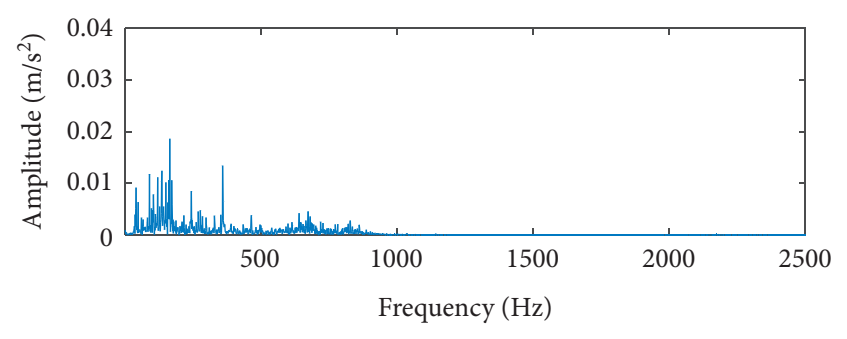

(a)

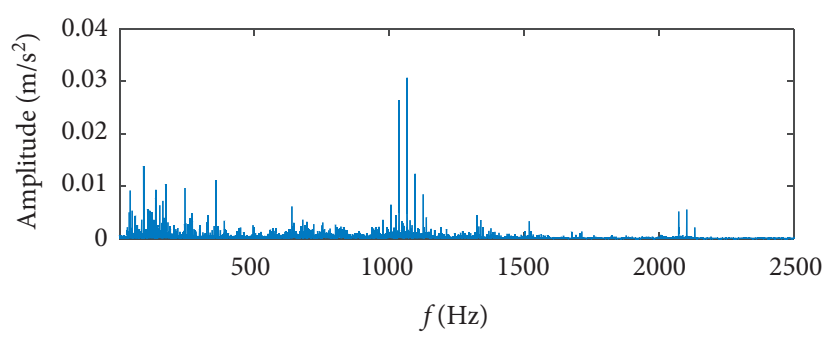

(b)

FiguRE 7: The spectrum of vibration signal (a) after wavelet denoising and (b) after SCICA-wavelet denoising.

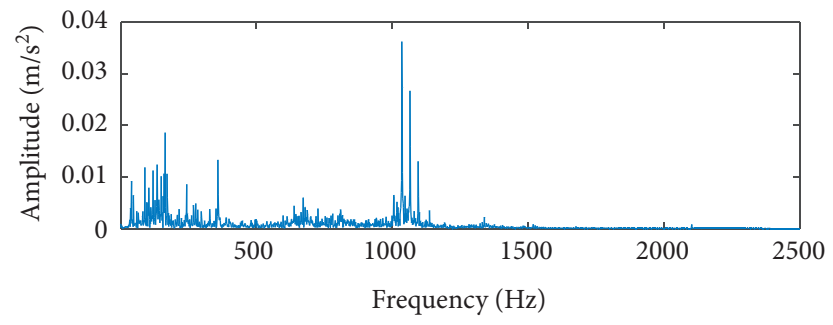

(a)

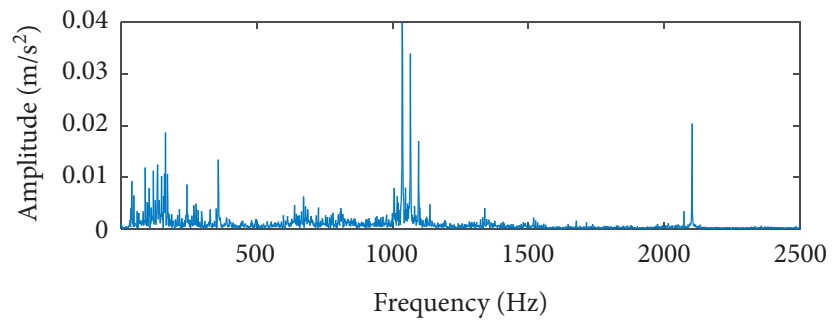

(b)

FIGURE 8: The spectrum of vibration signal (a) using smooth filtering and (b) using SCICA-FD with smooth filtering.

and thus,

$$
\sum_{k=0}^{p} a_{k} \sum_{i=-M}^{M} i^{k+r}=\sum_{i=-M}^{M} x(i) i^{r}
$$

Let

$$
\begin{aligned}
F_{r} & =\sum_{i=-m}^{M} x(i) i^{r}, \\
S_{k+r} & =\sum_{i=-M}^{M} i^{k+r},
\end{aligned}
$$

and then, equation (15) can be written as

$$
F_{r}=\sum_{k=0}^{P} a_{k} S_{k+r}
$$

Given the number of point $M$ needed to fit, polynomial order $P$, and the data $x(i), i=M, \ldots, 0, \ldots, M$, to be fitted, the coefficients $a_{0}, a_{1}, \ldots, a_{p}$ can be calculated by equation (17) and thus get the polynomial $f_{i}$.

In the actual calculation, the best fitting polynomial to center point $x(0)$ can be obtained as long as the coefficient $a_{0}$ is obtained, and assume $M=2$ and $P=2$, and applying equation (17), we obtain

$$
a_{0}=\left[-2 x(-2)+12 x(-1)+17 x(0)+12 x(1)-\frac{3 x(2)}{35}\right] \text {, }
$$

and consider $a_{0}$ as a filter factor or template:

$$
h(n)=\frac{[-3,12,17,12,-3]}{35} .
$$

Moving the template on data $x(n)$, central point $f_{0}$ can be calculated according to equation (19) and thus achieve fitting of $x(n) . h(n)$ is a symmetric FIR filter, which is $n=O$ symmetry, so it has the characteristics of zero phase, and its coefficients sum equals 1 , and its frequency characteristic is as follows:

$$
H\left(\frac{e^{j w}=[17+24 \cos w-6 \cos (2 w)]}{35}\right) .
$$

The smooth filter used in this paper set is $M=5$ and $p=3$.

3.3.2. SCICA-FD with Smooth Filtering. Using the same data, recall that the time domain signal is plotted in Figure 5(a) and its spectrum is plotted in Figure 5(b). Apart from the denoising method, nothing changes, and compare $d$ to experiment 2 . That is, the SCICA-FD output is the same, and the fractal dimension of each separated signal is the same as that in Figure 6.

The spectrum of the restored signal is shown in Figure 8(b). Applying only smooth filtering on the vibration signal, the filtering result is shown in Figure 8(a). After smooth filtering, the spectrum is similar to that of wavelet denoising as both algorithms have the characteristic of lowpass filter. First, carry out the proposed SCICA-FD than filtering only part of the component signals, and the result is shown in Figure 8(b). Again, both the $1000 \mathrm{~Hz}$ and $2000 \mathrm{~Hz}$ high frequency are retained and frequency elsewhere is depressed. From Figure 8(b), it is clear that the spectrum of vibration signal using SCICA-FD with smooth filtering has obvious fault shock characteristics at $1000 \mathrm{~Hz}$ and $2000 \mathrm{~Hz}$. 


\section{Conclusion}

The experimental results demonstrate that vibration signal components are complex. The signals exhibit both nonlinear and nonstationary characteristics. The proposed vibration noise reduction method combines the advantages of fractal analysis, blind source separation, and different denoising algorithms. Using the fractal dimension constraint with the SCICA, the denoising process avoids filtering useful information. Thus, the proposed method can reduce noise while ensuring that useful information is retained. The signal after noise reduction is smoother, and the burr is reduced. Although the amplitude of the noise was significantly attenuated because of the ICA algorithm, this change does not affect the analysis of the frequency domain components because changes in amplitude do not affect the time-frequency distribution. The proposed method effectively reduces noise and may have many theoretical and practical applications. Future work may show that the proposed method may be extended to other areas of research in which noise reduction is required.

\section{Data Availability}

The data used to support the findings of this study are available from the corresponding author upon request.

\section{Conflicts of Interest}

The authors declare that they have no conflicts of interest.

\section{Acknowledgments}

This work was financially supported by the National Natural Science Foundation of China (Grant nos. 41374185 and 415572347). The authors appreciate the support of project NYB17201600300 of State Key Laboratory of Operation and Control of Renewable Energy \& Storage Systems, China Electric Power Research Institute. Beijing 100192, China.

\section{References}

[1] Z. Qin, L. Chen, and X. Bao, "Wavelet denoising method for improving detection performance of distributed vibration sensor," IEEE Photonics Technology Letters, vol. 24, no. 7, pp. 542-544, 2012.

[2] Z. J. Xie, B. Y. Song, Y. Zhang, and F. Zhang, "Application of an improved wavelet threshold denoising method for vibration signal processing," Advanced Materials Research, vol. 889-890, pp. 799-806, 2014.

[3] M. Li, X. Wu, and X. Liu, "An improved EMD method for time-frequency feature extraction of telemetry vibration signal based on multi-scale median filtering," Circuits, Systems, and Signal Processing, vol. 34, no. 3, pp. 815-830, 2015.

[4] Y. X. Li, L. Wang, X. P. Li, and X. O. Yang, "A novel linear spectrum frequency feature extraction technique for warship radio noise based on complete ensemble empirical mode decomposition with adaptive noise, duffing chaotic oscillator, and weighted-permutation entropy," Entropy, vol. 21, no. 507, pp. 1-17, 2019.
[5] Y.-X. Li and L. Wang, "A novel noise reduction technique for underwater acoustic signals based on complete ensemble empirical mode decomposition with adaptive noise, minimum mean square variance criterion and least mean square adaptive filter," Defence Technology, vol. 16, no. 3, pp. 543-554, 2020.

[6] Z. J. Wang, N. N. Yang, N. P. Li, W. H. Du, and J. Y. Wang, "A new fault diagnosis method based on adaptive spectrum mode extraction," Structural Health Monitoring, pp. 1-17, 2021.

[7] Z. Wang, W. Zhao, W. Du, N. Li, and J. Wang, "Data-driven fault diagnosis method based on the conversion of erosion operation signals into images and convolutional neural network," Process Safety and Environmental Protection, vol. 149, pp. 591-601, 2021.

[8] A. Hyvärinen and E. Oja, "Independent component analysis: a tutorial," IEEE Transactions on Neural Networks, vol. 15, no. 2, p. 529, 2009.

[9] C. J. James and D. Lowe, "Single channel analysis of electromagnetic brain signals through ICA in a dynamical systems framework," in Proceedings of the 23rd Annual EMBS International Conference, pp. 1974-1977, Istanbul, Turkey, 2001.

[10] M. E. Davies and C. J. James, "Source separation using single channel ICA," Signal Processing, vol. 87, no. 8, pp. 1819-1832, 2007.

[11] M. Congedo, C. Gouy-Pailler, and C. Jutten, "On the blind source separation of human electroencephalogram by approximate joint diagonalization of second order statistics," Clinical Neurophysiology, vol. 119, no. 12, pp. 2677-2686, 2008.

[12] G. R. Naik, S. E. Selvan, and H. T. Nguyen, "Single-channel EMG Classification with ensemble-empirical-mode-decomposition-based ICA for diagnosing neuromuscular disorders," IEEE Transactions on Neural Systems and Rehabilitation Engineering, vol. 24, no. 7, pp. 734-743, 2016.

[13] W. Dai, D. Huang, and B. Liu, "A phase space reconstruction based single channel ICA algorithm and its application in dam deformation analysis," Survey Review, vol. 47, no. 345, 2015.

[14] C. Guerrero-Mosquera and A. N. Vazquez, "Automatic removal of ocular artifacts from EEG data using adaptive filtering and independent component analysis," in Proceedings of the 17th European Signal Processing Conference, pp. 2317-2321, Glasgow, Scotland, 2009.

[15] M. A. Klados, C. Papadelis, C. Braun, and P. D. Bamidis, "REG-ICA: a hybrid methodology combining blind source separation and regression techniques for the rejection of ocular artifacts," Biomedical Signal Processing and Control, vol. 6, no. 3, pp. 291-300, 2011.

[16] N. H. Packard, J. P. Crutchfield, J. D. Farmer, and R. S. Shaw, "Geometry from a time series," Physical Review Letters, vol. 45, no. 9, pp. 712-716, 1980.

[17] S. P. Arjunan, D. K. Kumar, and K. Dinesh, "Fractal properties of surface electromyogram for classification of low-level hand movements from single-channel forearm muscle activity," Journal of Mechanics in Medicine and Biology, vol. 11, no. 3, pp. 581-590, 2011.

[18] A. Phinyomark, P. Phukpattaranont, and C. Limsakul, "Fractal analysis features for weak and single-channel upperlimb EMG signals," Expert Systems with Applications, vol. 39, no. 12, pp. 11156-11163, 2012.

[19] F. Takens, Detecting Strange Attractors in Turbulence, Vol. 898, Springer, Groningen, Netherlands, 1980.

[20] J. Y. Yang, Y. Y. Zhang, Y. S. Zhu, and Q. H. Wang, "Intelligent fault diagnosis of rolling element bearing based on 
svms and statistical characteristics," in Proceedings of the ASME 2007 International Manufacturing Science and Engineering Conference, pp. 525-536, Atlanta, GA, USA, 2007.

[21] Y. Li, Z. Ma, W. Lu, and Y. Li, "Automatic removal of the eye blink artifact from EEG using an ICA-based template matching approach," Physiological Measurement, vol. 27, no. 4, pp. 425-436, 2006.

[22] S. M. Li, "Blind source separation of rotor vibration faults," Journal of Aerospace Power, vol. 20, no. 5, pp. 751-756, 2005.

[23] D. L. Donoho, "Denoising via soft thresholding," IEEE Transactions on Information Theory, vol. 41, no. 3, pp. 613627, 1992.

[24] A. Savitzky and M. J. E. Golay, "Smoothing and differentiation of data by simplified least squares procedures," Analytical Chemistry, vol. 36, no. 8, pp. 1627-1639, 1964. 